\title{
DISEÑO Y EVALUACIÓN DE UNA INTERVENCIÓN DIDÁCTICA BASADA EN APRENDIZAJE MÓVIL PARA MEJORAR LA IO DE ESTUDIANTES DE ELE
}

Mercedes Idalith León Axelsson

UNED

idalithl@hotmail.com

\section{RESUMEN}

El trabajo que aquí se presenta tiene como objetivo describir el diseño y la evaluación de una intervención didáctica basada en aprendizaje móvil (IDAM) y desarrollada con el fin de contribuir a mejorar la destreza de interacción oral (IO) de aprendices de español como lengua extranjera (ELE). Se inicia el artículo con una breve presentación del estado de esta área de investigación, a continuación se introduce el marco pedagógico de referencia y se describe el diseño mismo de la IDAM. Se presentan luego, sucintamente, los objetivos y el método del proceso de evaluación y, de manera detallada, los resultados de la misma. Se cierra el artículo con las conclusiones generales.

Los aportes centrales del estudio están referidos, por una parte, a la efectividad del aprendizaje móvil para mejorar la destreza de expresión oral, área de investigación aún incipiente, y por otra, a las reacciones de los alumnos frente a la innovación educativa. La evaluación realizada muestra que la IDAM fue percibida como una forma de aprendizaje efectiva para desarrollar la destreza de IO. También pone en evidencia que, si bien esta innovación educativa implicó algunos retos y dificultades, se percibió como una experiencia positiva, entretenida y enriquecedora.

PalABRAs Clave: aprendizaje de lenguas asistido por dispositivos móviles; Interacción Oral; aprendizaje de ELE; innovación educativa en lenguas.

\section{ABSTRACT}

The purpose of the work presented here is to describe the design and the assessment of an instructional module that, based on mobile learning (IMML), was developed in order to improve the oral interaction of students of Spanish as a foreign language.

A brief overview, regarding previous research into mobile assisted language learning, specifically aimed at developing speaking skills, is presented at the beginning this paper. The pedagogical frame of reference, followed by a description of the design of the instructional module, is outlined as well. The objectives, method and results of assessing this module are presented afterwards. The article is closed with the general conclusions. 
The main contributions of this study are to show the effectiveness of mobile learning for improving verbal communication skills as well as the students' reactions to this type of educational innovation. The assessment showed that the students perceived IMML as an adequate methodology to develop their oral skills. It also showed that despite the fact that working with this module entailed some difficulties and was a challenge; it was above all a positive and instructive experience.

KeYwords: Mobile Assisted Language Learning MALL; oral interaction; Spanish as a foreign language; educational innovation in languages.

\section{INTRODUCCIÓN}

Este artículo está relacionado con dos grandes áreas del conocimiento. Una de ellas es la didáctica de las lenguas extranjeras y, de forma más específica, la didáctica de la expresión oral que, a su vez, incluye como componente básico la IO. La segunda área de conocimiento viene de las TIC (tecnologías de la comunicación y la información) y se trata del aprendizaje de lenguas asistido por dispositivos móviles o MALL (Mobile Assisted Language Learning). Estas dos áreas del conocimiento son relativamente nuevas y presentan muchos retos didácticos y de investigación. El trabajo que se presenta a continuación es un intento por integrar dichas áreas, mediante el diseño de una intervención didáctica basada en aprendizaje móvil (IDAM) para desarrollar la destreza de IO de estudiantes de ELE.

En lo referente al diseño de la IDAM, podemos decir que es un ejercicio bastante interesante y exigente en los primeros intentos. Como menciona Kukulska-Hulme et al. (2015), la tendencia a repetir lo que generalmente se hace en clase es fuerte; por este motivo, el uso de un marco de referencia como el que se usó en el caso que aquí se describe resulta de gran ayuda. Por una parte, porque facilita la inclusión de aspectos que, de no tener suficiente experiencia en el diseño de este tipo de intervenciones, se pueden dejar de lado fácilmente. Ejemplos de ello son la evaluación por pares o la reflexión sobre el propio aprendizaje. Por otra parte, un marco de referencia alusivo específicamente al diseño de una unidad de aprendizaje móvil promueve la reflexión sobre las funciones que potencialmente tienen los dispositivos digitales y, en consecuencia, facilita el uso consciente de tales funciones en el diseño de dicha unidad móvil.

Como resulta evidente, estas dos posibilidades contribuyen a que el contacto del aprendiz con la lengua objeto sea lo más cercano posible a la realidad, factor definitivo para el aprendizaje de una lengua, según los dos enfoques asumidos como base para el diseño de esta IDAM: el enfoque comunicativo (Wilkins, 1976; Littlewood, 1981; Melero, 2008) y el aprendizaje por tareas (Nunan, 1996; Willis \& Willis, 2007).

También es interesante examinar lo que implica incorporar la característica esencial de este tipo de aprendizaje, es decir, la movilidad de los dispositivos, del aprendizaje y de los aprendices. No se debe desconocer que factores tales como el acceso a una conexión de internet, la familiaridad con el uso de los dispositivos y los recursos móviles, la edad y el estilo de aprendizaje, entre otros, influyen de manera directa en el éxito de cada expe- 
riencia de aprendizaje móvil (Deegan, 2015; Sung, Chang \& Yang, 2015; Shuler, Winters \& West, 2013). En el caso particular de la IDAM que aquí se presenta, se quiso incorporar la movilidad de una manera modesta, un tanto controlada, puesto que se diseñó para ser incorporada al currículum de las clases regulares presenciales de ELE en la enseñanza básica secundaria, contexto en el cual la autonomía de los estudiantes, al igual que su familiaridad con el uso de las tecnologías móviles en entornos de aprendizaje es limitada. Así, los dispositivos móviles utilizados fueron principalmente computadores portátiles y tabletas, dejando el uso de teléfonos móviles a la voluntad de los participantes. Por su parte, la movilidad de los aprendices estuvo pensada sobre todo en el espacio escolar, por cuanto la IDAM se desarrolló principalmente durante las horas destinadas a la clase de ELE, aunque los aprendices tuvieron la posibilidad de interactuar con ella en cualquier tiempo y espacio. Lo mismo se aplica a la movilidad del aprendizaje, pues, aunque potencialmente, se podría aprender desde cualquier sitio en el que los participantes tuvieran acceso a internet, se previó que se llevaría a cabo ante todo en los espacios escolares y en la casa de los aprendices, como efectivamente se corroboró por medio de los instrumentos de evaluación.

Con el fin de ubicar este trabajo en el contexto de la literatura pertinente se presenta inicialmente el estado de la cuestión para luego discutir las características de MALL y sus implicaciones pedagógicas. Enseguida se presentan los fundamentos metodológicos que subyacen a esta intervención didáctica y se discute su diseño. Se describe, finalmente, el proceso de evaluación, incluyendo los objetivos, el método y los resultados.

\section{ESTADO DE LA CUESTIÓN}

Diversos meta-análisis (Sung, Chang \& Yang, 2015; Burston, 2014; Viberg \& Grönlund, 2013; Demouy \& Kukulska-Hulme, 2010) sobre el uso de MALL durante las dos últimas décadas indican que los estudios que tratan específicamente el asunto del desarrollo de la expresión oral no son numerosos. A continuación se reseñan los más recientes (de 2013 a 2015), haciendo énfasis en los resultados alcanzados y en el aspecto lingüístico investigado.

El primero de ellos es el trabajo de Moladoust (2014), en el cual 15 estudiantes iraníes de ELE utilizaron Diarios de Grabaciones de Diálogos Orales o ATODJs (Audiotaped Oral Dialogue Journals), como parte de su proceso de aprendizaje. Este método, introducido por Lanzaraton (2001), consiste en la grabación de producciones orales que los estudiantes realizan como una tarea extraescolar, a partir de un tema que ha sido introducido por el profesor. Tales grabaciones semanales deben ser espontáneas y deben tener como foco la fluidez, el intercambio de significados y la corrección lingüística. Una vez realizadas, son entregadas al profesor, quien las revisa y brinda inmediata retroalimentación individual a los estudiantes, a partir de sus necesidades y sobre elementos específicos acordados (Moladoust, 2014). Mediante métodos cualitativos se encontró que tanto los profesores como los estudiantes participantes en este programa, consideraron que los ATODJs fueron de gran ayuda para el desarrollo de la destreza oral (íbid).

Gromik (2015) avanza en la propuesta de utilizar la grabación regular de vídeos como apoyo para el aprendizaje del inglés, experimentando con la producción de Digital Stories, 
es decir, historias filmadas con la cámara del teléfono móvil. En este estudio de caso, 67 estudiantes de EFL (English as a Foreign Language) de la Universidad Tohoku de Japón produjeron un vídeo semanal de 30 segundos con la cámara de su móvil, durante 12 semanas, partiendo de un tema propuesto por el profesor pero que ellos deberían adaptar, delimitar y orientar. Los resultados de este estudio mostraron que los estudiantes pudieron producir videos con historias digitales para expresar sus opiniones, y que esta tarea fue percibida como relevante para el aprendizaje de la segunda lengua.

Un tercer estudio contemporáneo que se refiere a la mejora de la destreza oral, es el adelantado en Corea por Ahn \& Lee (2015). Este trabajo tuvo como objetivo analizar las experiencias y percepciones de los usuarios de Speaking English 60 Junior, un sistema con reconocimiento de voz para el aprendizaje del inglés como LE. Los resultados del experimento mostraron que los estudiantes tuvieron una actitud muy positiva hacia el uso de esta aplicación y valoraron sobre todo la función de reconocimiento de voz, en especial la posibilidad de interactuar oralmente con un personaje, ya que les permitía observar de manera inmediata y directa las consecuencias de sus producciones orales. Esta experiencia resulta especialmente interesante por cuanto tiene como foco de estudio la IO, objeto de la experiencia didáctica descrita en este artículo y aspecto lingüístico-comunicativo muy poco estudiado hasta la fecha.

Por último, se debe mencionar el estudio realizado en la Universidad Nacional de Educación a Distancia (UNED, España), sobre Vídeos para hablar o VISP (Videos for Speaking), una aplicación móvil que utiliza la audiodescripción (AD) para mejorar la competencia oral a diferentes niveles, mediante pequeños fragmentos de vídeo. (Ibáñez, Vermeulen \& Jordano, 2016). Los resultados de este estudio «permiten esbozar un marco teórico-pedagógico en el que se puede enmarcar una aplicación de MALL que se vaya a usar en la educación a distancia» (íbid, p. 2). Además, el estudio mostró que los estudiantes que evaluaron VISP como una herramienta adecuada para practicar la expresión oral a la vez que se desarrolla la competencia léxica, fueron estudiantes de educación a distancia, acostumbrados a utilizar las TIC; mientras que estudiantes de programas presenciales mostraron una motivación más baja hacia el uso de esta aplicación. (Ibáñez \& Vermeulen, en prensa).

A partir de los casos antes reseñados es posible afirmar que los intentos contemporáneos por realizar una innovación pedagógica para mejorar la expresión oral con el apoyo de recursos móviles, aunque no son muchos, muestran un avance en la concepción pedagógica que subyace a ellos. Además, en estos nuevos entornos de aprendizaje los dispositivos móviles se usan de manera más creativa, potencializando los recursos que ofrecen, y el maestro asume un rol más de guía que de productor de contenidos. Sin embargo, se necesitan desarrollar más experiencias innovadoras que sigan explorando las posibilidades pedagógicas del MALL y que arrojen luces sobre los hechos didácticos implicados, como esperamos sea el caso de la experiencia que se describe en este artículo.

\section{APRENDIZAJE MÓVIL: IMPLICACIONES PEDAGÓGICAS}

El vertiginoso desarrollo de las herramientas de comunicación digital ha dado lugar, en lo andado del siglo XXI, a un mundo globalizado donde la tecnología posibilita el llamado 
«aprendizaje móvil» que, en el contexto de aprendizaje de lenguas, se conoce como MALL (Mobile Assisted Language Learning). En palabras de Taylor (2005), una teoría del aprendizaje en una sociedad móvil como la del nuevo milenio

...encompasses both learning supported by mobile devices such as cellular (mobile) phones, portable computers and personal audio players, and also learning in an era characterized by mobility of people and knowledge (Rheingold, 2002) where the technology may be embedded in fixed objects such as walk up and use information terminals. For brevity we refer to these together as mobile learning. (Taylor, 2005: 3)

Es decir, el aprendizaje móvil se entiende como el aprendizaje soportado por dispositivos móviles, pero también como el aprendizaje que se da en una era caracterizada por la movilidad de los aprendices y del conocimiento, en pocas palabras, el aprendizaje que gracias a la hand-held mobile technology es posible en cualquier momento y en cualquier lugar.

Si bien esta definición es la más generalizada actualmente, cabe señalar que, durante los primeros años de exploración del MALL, los desarrollos y experiencias estuvieron centrados en el hecho de usar dispositivos móviles en procesos de aprendizaje de lenguas, sin que el interés se centrara en cómo usarlos. Efectivamente, las primeras aplicaciones de MALL tendieron a replicar actividades tradicionales dentro de la didáctica de las lenguas. Esta tendencia ha cambiado solamente en los últimos cinco años, cuando el énfasis se ha puesto más claramente en el cómo usar de forma innovadora, desde el punto de vista pedagógico, todas las posibilidades que ofrecen las características de las tecnologías móviles. Pues, como ya lo anunciaban Sharples, Taylor \& Vavoula (2007) en su intento por formular una teoría sobre el MALL, tales características requieren de una nueva perspectiva pedagógica que, aunque basada en enfoques pedagógicos ya conocidos, como el constructivismo, dé cabida a los nuevos elementos, conceptos y relaciones generados en este tipo de ambientes de aprendizaje. Esto mismo se aplica al caso de la enseñanza aprendizaje de LE donde las características y naturaleza del aprendizaje móvil, además de permitir potenciar al máximo los postulados del enfoque comunicativo, llevan a re-conceptualizar en varios aspectos las relaciones y elementos implicados en el proceso de enseñanza aprendizaje (Kukulska-Hulme, Norris \& Donohue, 2015).

Siguiendo nuevamente a autores como Deegan (2015), Sung, Chang \& Yang (2015) y Shuler, Winters \& West (2013), podemos afirmar que las características propias de un ambiente de aprendizaje móvil son principalmente: ubicuidad, conectividad social e interacción, sensibilidad al contexto, individualización, inmediatez y vertiginosidad. Como ya se mencionó, estas características tienen consecuencias potenciales en los componentes pedagógicos y didácticos de la enseñanza y el aprendizaje de las lenguas extranjeras y exigen una re-conceptualización de los mismos. Por ejemplo, si tomamos el rol de profesores y alumnos, podemos decir que nunca antes ha resultado tan evidente la posibilidad que tienen, tanto los unos como los otros, de ser participantes activos en el proceso de aprender una lengua, pues como lo señalan Kukulska-Hulme, Norris \& Donohue (2015) MALL incrementa la posibilidad que tienen los estudiantes de asumir la responsabilidad de su propio aprendizaje, y los profesores, de facilitar a sus alumnos este proceso a través del diseño de actividades y materiales adecuados que potencialicen al máximo los recursos digitales 
móviles, tanto dentro como fuera del salón de clase. Con el fin de ayudar a los profesores en esta tarea, Kukulska-Hulme et al. (2015) proponen el marco pedagógico de referencia ${ }^{1}$ que ha servido como base para diseñar la intervención didáctica que se presenta en este documento, lo cual es objeto del siguiente apartado².

\section{DISEÑO DE LA IDAM}

El diseño de una intervención didáctica para integrar el MALL en el aula de ELE requiere tener en cuenta aspectos que no se considerarían en otro tipo de diseños, ya que están dados por las características exclusivas de las tecnologías y recursos móviles mencionadas anteriormente. Según el marco de referencia propuesto por Kukulska-Hulme et al. (2015), los factores centrales que intervienen en el diseño de una actividad de MALL son cuatro: el saber del profesor, las características de los dispositivos, la movilidad de los aprendices y la dinámica del lenguaje.

Este marco de referencia propone incluir, además, cuatro conceptos conectores (connecting concepts). Ellos buscan ayudar al profesor a tomar conciencia de que las actividades planeadas y diseñadas para la modalidad de aprendizaje móvil deben ser diferentes a las actividades planeadas normalmente, con el fin de contribuir a una verdadera innovación y evitar así que se trate solamente de transferir viejas ideas a formatos nuevos.

Cada uno de estos conceptos incluye una pregunta guía para el profesor. Son varias las razones por las cuales estos cuatro conceptos conectores y las preguntas relacionadas con cada uno de ellos resultan de gran ayuda en el momento de diseñar una intervención didáctica que incorpore tecnologías móviles. En primer lugar, porque facilitan la puesta en práctica de principios pedagógicos propios de los enfoques contemporáneos sobre la enseñanza-aprendizaje. En segundo lugar, porque obligan a pensar más allá de los esquemas normalmente utilizados, lo cual a su vez lleva a re-conceptualizar algunos de los componentes involucrados en el proceso de aprender una lengua. También porque ayudan a mantener un hilo conductor entre las diferentes tareas y ejercicios propuestos. Por último, porque contribuyen a efectivizar y enriquecer el proceso de aprendizaje, ya que las preguntas planteadas llevan a escoger de manera muy consciente todas las actividades y tareas a desarrollar.

Podemos anotar, finalmente, que este marco de referencia permite reflejar la nueva dinámica de un aula en la que, quizá los alumnos y/o el profesor no están presentes físicamente, pero tienen un alto grado de interactividad. En este contexto, los estudiantes indagan, crean y comparten y el profesor motiva y guía. En una clase en el la que, como lo señala

\footnotetext{
${ }^{1}$ Este marco de referencia es el resultado de una investigación desarrollada por la Open University en 20132014 con financiación del Consejo Británico (Kukulska-Hulme et al., 2015: 2).

${ }^{2}$ Es importante mencionar que además de este MR, para el diseño y desarrollo de la IDAM se han tenido en cuenta los postulados teóricos y metodológicos provenientes de dos paradigmas de gran relevancia dentro de la enseñanza y aprendizaje de lenguas: el Enfoque Comunicativo, en inglés Communicative Language Teaching (CLT) (Wilkins, 1976) y el Aprendizaje por Tareas o Task-Based Learning (TBL) (Prabhu, 1987; Breen, 1987; Candlin, 1990; Nunan, 1989; Wllis \& Willis, 2007).
} 
Pegrum (2014), tanto el profesor como los alumnos reconocen los beneficios de la construcción del conocimiento y de la creación de redes colaborativas.

Con base en este marco de referencia se diseñó la IDAM que busca mejorar la destreza de IO en alumnos de ELE con un nivel de lengua A2, según a los criterios del Marco Común Europeo de Referencia para la Enseñanza y Evaluación de las Lenguas MCER ${ }^{3}$ (Consejo de Europa, 2002). Se escogió este componente de la expresión oral por cuanto en un estudio piloto se identificó ésta como una de las principales necesidades de los aprendices. Además, porque a pesar de ser tan fundamental en la comunicación, son pocos los estudios que se han hecho acerca de su enseñanza y aprendizaje, tanto en el marco de la didáctica de LE como del MALL.

Veamos de qué forma se integraron a la IDAM los cuatro factores centrales y los cuatro conceptos conectores planteados en el marco de referencia antes descrito.

En primer lugar, el saber del profesor (teacher wisdom) está involucrado en el diseño de la IDAM por cuanto es el docente quien selecciona el tema central de aprendizaje y quien diseña las tareas, con base en su conocimiento sobre las características y necesidades del grupo, a partir de unos postulados teóricos y metodológicos concretos. Además, su rol durante todo el proceso es claramente de orientador, pues guía a los alumnos a lo largo del desarrollo de la IDAM y les proporciona continua retroalimentación sobre las diferentes tareas que van realizando. Este rol se facilita gracias a recursos propios de los dispositivos móviles, como las aplicaciones de chat y los mensajes compartidos o privados.

En cuanto al segundo factor, las funciones de los dispositivos (device features), se incorporan de diversas maneras. Por una parte, los dispositivos móviles se utilizan como monitores para mostrar información multimodal (texto, audios, vídeos), como por ejemplo, las explicaciones de la profesora, los audios modelos de pronunciación y los vídeos de diálogos auténticos. Además, se usan como herramientas de grabación y de reflexión, por cuanto los estudiantes adelantan procesos metacognitivos al intuir, definir, comparar y analizar las estrategias de IO utilizadas en las charlas modelo, en relación con sus propias charlas y las de sus compañeros de clase. Estos procesos se llevan a cabo mediante la resolución de guías, la producción de comentarios y la creación de un diario digital en el que los alumnos registran sus reflexiones sobre el trabajo realizado.

Por último, en el contexto extraescolar, además de tener acceso a las funciones antes descritas, los estudiantes tienen la posibilidad de interactuar con hablantes nativos mediante vídeo y audio.

Respecto a las movilidades de los aprendices (learner mobilities), es decir, los lugares y tiempos en los que ellos pueden aprender, están conformadas principalmente por la escuela y la casa, aunque potencialmente el aprendizaje puede tener lugar en cualquier tiempo y espacio.

Por otra parte, el nivel de motivación, elemento también importante en la movilidad de los aprendices, se pone a prueba al proponerles a ellos actividades extraescolares, como la

${ }^{3}$ El Marco Común Europeo de Referencia para el aprendizaje, la enseñanza y evaluación de lenguas, desarrollado por el Consejo de Europa, se constituye en la referencia internacional más aceptada oficialmente en lo referido a la enseñanza-aprendizaje de L2. Según sus estándares, el nivel A2 corresponde al nivel de usuario básico. (Consejo de Europa, 2002) 
automatización de elementos léxicos o la realización de una entrevista. Así mismo, se debe considerar que el propósito individual con el que cada aprendiz desarrolla las tareas propuestas juega un papel decisivo en el aprendizaje.

En cuanto al último de los factores, la dinámica del idioma (language dynamics), queda abierta la posibilidad de que los significados y cambios de la lengua sean analizados a partir de las diferentes tareas que implican procesos de interacción y de reflexión y que se realizan por medio de diferentes canales.

Veamos ahora cómo se concretan en la creación de la IDAM estos factores y los conceptos conectores. En primer lugar, debe decirse que el componente central de esta intervención didáctica es una unidad digital desarrollada por medio del programa VoiceThread (www.voicethread.com) ${ }^{4}$. Aunque integrada a las clases regulares del ELE, la IDAM está pensada para ser desarrollada de manera independiente por los estudiantes, es decir, para que sean ellos quienes asuman el control del acceso a los contenidos, del desarrollo de las tareas y del manejo de la interacción, tanto con sus compañeros de clase como con el profesor. Todo esto con el apoyo de dispositivos y recursos móviles.

Como muestra la figura 1, la IDAM se puede considerar como una red compuesta por diferentes nodos con conexiones ya establecidas, pero también con conexiones potenciales, que se generan por acciones de los aprendices.

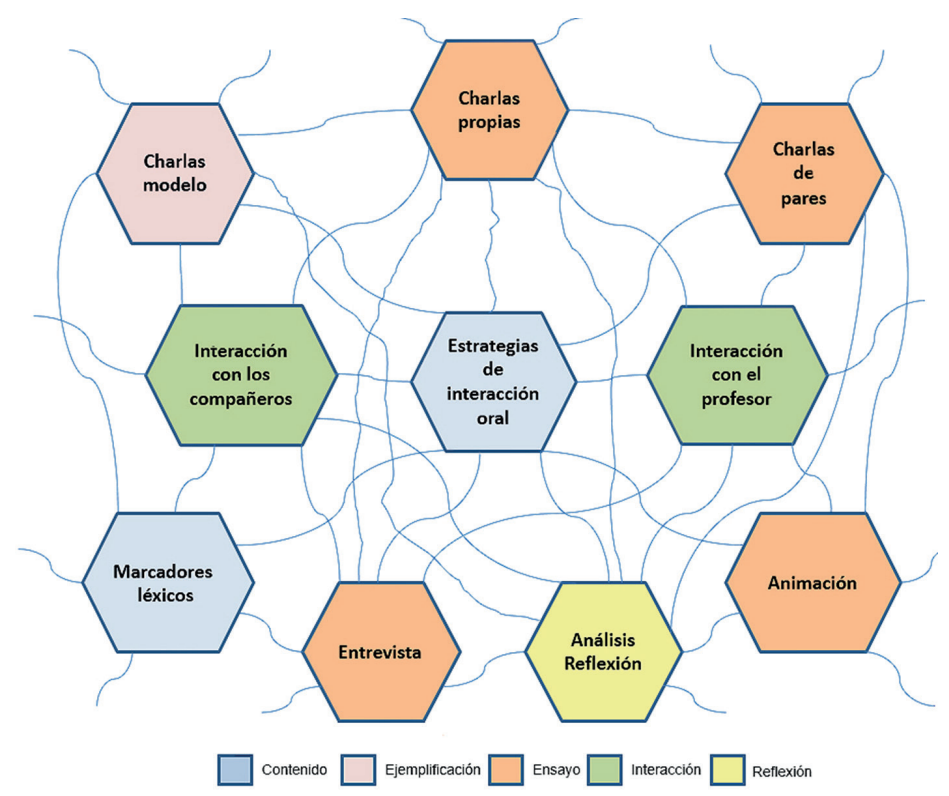

Figura 1. Nodos componentes de la IDAM.

${ }_{4}$ VoiceThread es una aplicación en línea que permite generar presentaciones interactivas, multimodales y colaborativas, gracias a los diferentes recursos que ofrece, tales como la inserción de todo tipo de archivos, de enlaces y comentarios. 
Existen cinco tipos de nodos: de contenido, de ejemplificación, de ensayo, de interacción y de reflexión. Los nodos de contenido son dos: el que presenta las seis estrategias de IO y el que presenta los marcadores léxicos de dichas estrategias. Las estrategias incluidas son : 1) iniciar la charla, 2) cambiar de tema, 3) finalizar la charla, 4) cooperar mediante comentarios, 5) cooperar mediante preguntas de seguimiento y 6) pedir aclaraciones. En este nodo los aprendices tienen la posibilidad de escuchar y leer una explicación sobre las seis estrategias orales por trabajar y sus respectivos marcadores léxicos para familiarizarse con su significado, escritura, pronunciación y uso en un contexto dado.

En el nodo de ejemplificación, como su nombre indica, los aprendices encuentran modelos de charlas con diferentes niveles de interacción; en ellas pueden identificar las estrategias de IO que se han descrito en el nodo de contenido. Se espera que los estudiantes usen estas charlas como referencia para desarrollar las suyas propias en los nodos de ensayo.

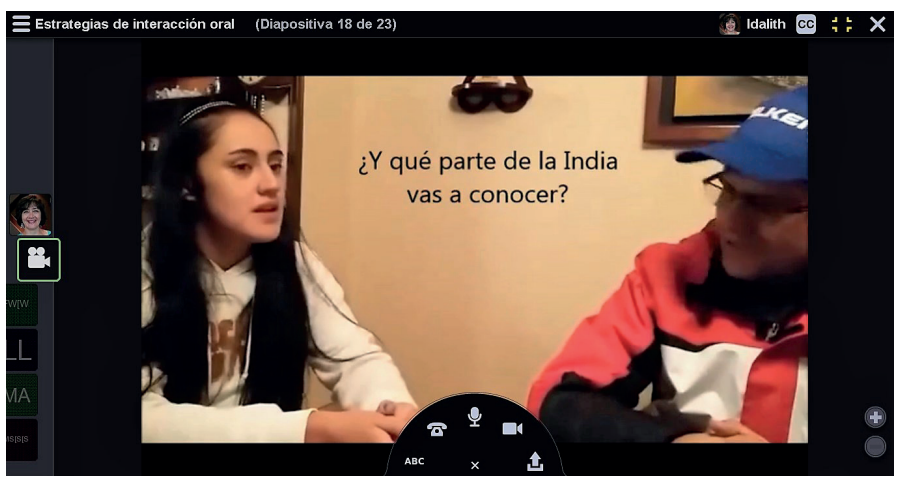

Figura 2. NCharla en VoiceThread.

La figura 2 muestra una de las charlas presentadas en VoiceThread como modelo de una conversación con alto nivel de interactividad. Una de las tareas asignadas a los estudiantes fue la de escuchar esta charla, analizarla e identificar las estrategias de IO usadas por los hablantes, así como los marcadores léxicos correspondientes a cada estrategia de IO.

Por su parte, los nodos de interacción contienen las tareas conducentes a que los aprendices se comuniquen entre sí o con el profesor. En la figura vemos que se incluyen dos nodos de este tipo, uno de interacción entre los aprendices y otro entre ellos y el profesor. En el primer caso, la interacción posibilita el trabajo colaborativo entre los estudiantes con el fin de resolver problemas e intercambiar información y retroalimentación; en el segundo caso, posibilita la comunicación permanente con el profesor para resolver dudas, obtener guía o recibir retroalimentación.

Cabe aclarar aquí que aunque la IDAM se desarrolló durante el tiempo asignado a las clases regulares de ELE, la profesora no estaba físicamente presente y, por lo tanto, su comunicación con los estudiantes se llevó a cabo a través de los dispositivos móviles. Estos procesos de interacción fueron posibles gracias a la función de mensajería multimodal de los recursos móviles usados, que permitió producir comentarios o enviar mensajes a un individuo o a todo el grupo. 
Volviendo a la figura 1, se puede observar que los nodos predominantes en la IDAM son los de ensayo, que corresponden a las tareas propiamente comunicativas. Para desarrollar cada una de ellas se utilizan diferentes recursos digitales ofrecidos por aplicaciones como la plataforma de aprendizaje Fronter, la red social de Facebook y la aplicación VoiceThread. Estos nodos le ofrecen al aprendiz la posibilidad de aplicar los nuevos conocimientos de diferentes maneras; por ejemplo creando y grabando charlas cortas, construyendo una animación digital o entrevistando a un hablante nativo.

Por último, el nodo de reflexión reúne las tareas que permiten generar en los participantes procesos metacognitivos lingüísticos y pedagógicos. Los procesos de metacognición lingüística tienen que ver con la concienciación sobre la existencia y el uso de las estrategias de IO. Los procesos metacognitivos de carácter pedagógico se refieren a la reflexión de los estudiantes sobre su propio proceso de aprendizaje.

Para cerrar esta descripción de los nodos que componen la IDAM, se presentan, en la tabla 1, las diferentes tareas por realizar, así como su propósito y los recursos móviles utilizados para desarrollarlas. También se indica qué nodos están implicados en cada tarea.

En esta tabla también se puede ver que se incluyen tareas tanto posibilitadoras como comunicativas (Estaire y Zanón, 1990). Dentro de las tareas posibilitadoras están las referidas a la identificación de las estrategias de IO y a la automatización de marcadores léxicos. Las tareas propiamente comunicativas son las relacionadas con la producción de diálogos a manera de ensayo, así como la realización de una entrevista en línea. La IDAM incluye, además, una tarea final (íbid) que consiste en sostener una charla sobre temas conocidos, en la que se usen las estrategias de IO aprendidas. Mediante el desarrollo de estas tareas se incorporan los principios básicos que según Richards \& Rodgers (2010) fueron punto de partida para el diseño instruccional del aprendizaje de las lenguas propuesto por el enfoque comunicativo, a saber: el principio de comunicación, el principio de acción y el principio de significatividad.

El principio de comunicación está incorporado en la medida en que las tareas desarrolladas durante la intervención didáctica involucran situaciones reales de comunicación, como es el caso de sostener una charla en la lengua objeto para obtener información personal, o realizar una entrevista a un hablante nativo, con el mismo fin. El principio de acción, está incorporado por cuanto los aprendices han de usar la lengua objeto para realizar las tareas propuestas. Por último, también está presente el principio de significatividad, ya que al realizar actividades que tengan sentido para los aprendices, el lenguaje usado también se vuelve significativo (Ausbel, 1963).

Siguiendo nuevamente los postulados del enfoque comunicativo, para el desarrollo de algunas de las tareas incluidas en la IDAM, se prioriza el uso de material auténtico, tanto con el fin de exponer al aprendiz a la lengua real, como para brindarle la posibilidad de que desarrolle estrategias para comprenderla. En consecuencia, las charlas que sirven como punto de partida para el análisis de la IO fueron realizadas por hablantes nativos y se ajustaron en lo posible a una charla espontánea. 
Tabla 1. Tareas incluidas en la IDAM, propósito de cada una y recursos digitales utilizados para desarrollarlas

\begin{tabular}{|c|c|c|c|}
\hline NODOS & TAREA & PROPÓSITO & RECURSOS \\
\hline $\begin{array}{l}\text { Ejemplificación } \\
\text { Reflexión } \\
\text { Interacción }\end{array}$ & $\begin{array}{l}\text { Ver y comparar las charlas } \\
\text { auténticas presentadas en } \\
\text { dos vídeos. }\end{array}$ & $\begin{array}{l}\text { Determinar la diferencia entre ellos. } \\
\text { Compartir conclusiones, comentarios } \\
\text { y/o preguntas. }\end{array}$ & $\begin{array}{l}\text { VoiceThread } \\
\text { Facebook }\end{array}$ \\
\hline Reflexión & $\begin{array}{l}\text { Ver nuevamente el segundo } \\
\text { vídeo y analizarlo. }\end{array}$ & $\begin{array}{l}\text { Deducir las estrategias usadas por los } \\
\text { hablantes para mantener una charla } \\
\text { interactiva y compartir conclusiones. }\end{array}$ & $\begin{array}{l}\text { VoiceThread } \\
\text { Facebook }\end{array}$ \\
\hline $\begin{array}{l}\text { Contenido } \\
\text { Reflexión }\end{array}$ & $\begin{array}{l}\text { Reconocer algunos marca- } \\
\text { dores léxicos de las estra- } \\
\text { tegias de } 10 \text { e identificar los } \\
\text { nuevos. }\end{array}$ & $\begin{array}{l}\text { Concientizarse sobre la función de } \\
\text { algunos marcadores léxicos ya cono- } \\
\text { cidos y de otros nuevos, en relación } \\
\text { con las estrategias de IO. }\end{array}$ & $\begin{array}{l}\text { Fronter } \\
\text { VoiceThread }\end{array}$ \\
\hline Contenido & $\begin{array}{l}\text { Escuchar los audios corres- } \\
\text { pondientes a los marcado- } \\
\text { res léxicos identificados y } \\
\text { grabar la propia reproduc- } \\
\text { ción de los mismos. }\end{array}$ & $\begin{array}{l}\text { Entrenar la pronunciación, entona- } \\
\text { ción y significado. } \\
\text { Empezar a automatizar los nuevos } \\
\text { marcadores léxicos. }\end{array}$ & $\begin{array}{l}\text { Grabadora de so- } \\
\text { nido de los dispo- } \\
\text { sitivos móviles. } \\
\text { VoiceThread }\end{array}$ \\
\hline Reflexión & $\begin{array}{l}\text { Hacer una categorización } \\
\text { de los marcadores léxicos } \\
\text { revisados, de acuerdo a la } \\
\text { estrategia de } 10 \text { a la que } \\
\text { pertenecen. }\end{array}$ & $\begin{array}{l}\text { Reforzar el proceso metacognitivo } \\
\text { sobre las estrategias de IO y sus mar- } \\
\text { cadores léxicos. }\end{array}$ & Fronter \\
\hline Contenido & $\begin{array}{l}\text { Entrenar los marcadores lé- } \\
\text { xicos de las estrategias de } \\
\text { IO. }\end{array}$ & $\begin{array}{l}\text { Automatizar los marcadores léxicos } \\
\text { de las estrategias de IO. }\end{array}$ & $\begin{array}{l}\text { Quia } \\
\text { Glosboken } \\
\text { Kahoot }\end{array}$ \\
\hline $\begin{array}{l}\text { Ejemplificación } \\
\text { Reflexión }\end{array}$ & $\begin{array}{l}\text { Ver tres charlas y analizar } \\
\text { las estrategias de } 10 \text { utiliza- } \\
\text { das en cada una. }\end{array}$ & $\begin{array}{l}\text { Reconocer las estrategias de } 10 \text { y sus } \\
\text { marcadores léxicos en contexto. }\end{array}$ & $\begin{array}{l}\text { VoiceThread } \\
\text { Fronter }\end{array}$ \\
\hline Ensayo & $\begin{array}{l}\text { Realizar conversaciones } \\
\text { cortas en las que usen las } \\
\text { estrategias de } 10 \text { y grabar al } \\
\text { menos una de ellas. }\end{array}$ & $\begin{array}{l}\text { Utilizar conscientemente las estrate- } \\
\text { gias de } 10 .\end{array}$ & VoiceThread \\
\hline Interacción & $\begin{array}{l}\text { Comentar algunos de los } \\
\text { diálogos realizados por los } \\
\text { compañeros. }\end{array}$ & $\begin{array}{l}\text { Reforzar el conocimiento adquirido } \\
\text { sobre las estrategias de IO de manera } \\
\text { colaborativa. }\end{array}$ & VoiceThread \\
\hline $\begin{array}{l}\text { Ensayo } \\
\text { Interacción }\end{array}$ & $\begin{array}{l}\text { Realizar un diálogo a través } \\
\text { de una animación y comen- } \\
\text { tar las realizadas por los } \\
\text { compañeros. }\end{array}$ & $\begin{array}{l}\text { Reforzar el conocimiento adquirido } \\
\text { sobre las estrategias de } 10 \text { de manera } \\
\text { colaborativa. }\end{array}$ & $\begin{array}{l}\text { GoAnimate } \\
\text { Facebook }\end{array}$ \\
\hline Ensayo & $\begin{array}{l}\text { Realizar una entrevista a un } \\
\text { hablante nativo y compartir } \\
\text { lo aprendido en la experien- } \\
\text { cia. }\end{array}$ & $\begin{array}{l}\text { Utilizar las estrategias de } 10 \text { en una } \\
\text { situación comunicativa real }\end{array}$ & $\begin{array}{l}\text { Skype } \\
\text { VoiceThread }\end{array}$ \\
\hline
\end{tabular}


De la descripción anterior se puede deducir que en el diseño de la IDAM convergen tanto postulados teóricos como principios metodológicos y criterios didácticos de carácter contemporáneo. Por este motivo pensamos que puede constituir un buen ejemplo de innovación en las aulas de ELE, así como de integración del aprendizaje móvil a las clases regulares de LE.

\section{EVALUACION DE LA IDAM}

La intervención didáctica antes descrita fue utilizada y evaluada por una población de 26 alumnos de ELE del grado noveno de educación básica secundaria en el colegio Hammarlunden (Suecia).

La IDAM se incorporó a las clases regulares de ELE que, siguiendo la normativa del Ministerio de Educación sueco, cubren ciento sesenta minutos a la semana. Los estudiantes trabajaron con la IDAM durante cuatro semanas.

El objetivo de esta evaluación fue el de identificar las percepciones de los estudiantes sobre el uso de esta innovación educativa. Con este fin, se aplicaron dos instrumentos: un cuestionario electrónico y cuatro entrevistas grupales. A continuación se presentan los resultados obtenidos a partir del análisis de los datos recogidos.

\subsection{PERCEPCIONES DE LOS ESTUDIANTES}

Mediante los instrumentos antes mencionados se recogió información acerca de las percepciones de los estudiantes que usaron la IDAM, respecto de los siguientes ítems:

- Qué tanto mejoraron las diferentes destrezas relacionadas con la expresión oral, a partir del trabajo con la IDAM.

- Cuál fue el nivel de efectividad de las diferentes tareas en relación con el objeto de aprendizaje, es decir, la mejora de la IO.

- Cuáles fueron logros de la IDAM.

- Cuáles fueron las dificultades de la IDAM.

Para indagar sobre el primer ítem, se les pidió a los aprendices que valoraran la mejora de las diferentes destrezas implicadas en la expresión oral, a partir del trabajo con la IDAM, en una escala Likert de 1 a 5. Los resultados se pueden ver en la figura 3.

En esta figura se puede observar que, según la percepción de los aprendices, el aspecto que más mejoró gracias al trabajo con la IDAM fue el número 5, correspondiente al uso de las estrategias de IO, con una media de 3,72. Esta percepción fue corroborada a través de las entrevistas grupales, en las que los aprendices reconocieron no tener conocimientos previos, al menos conscientes, acerca de la existencia de estrategias de IO; por lo tanto, consideraron el objeto de aprendizaje de la IDAM muy útil y manifestaron haberlo asimilado. Así lo dejan ver opiniones como las siguientes:

«Pude tomar conciencia de cosas que ya sabía, como por ejemplo iniciar y terminar una charla de manera apropiada». 
«Aprendí algo nuevo. Uno no sabía que había estrategias».

«Tuve la sensación de aprender mucho».

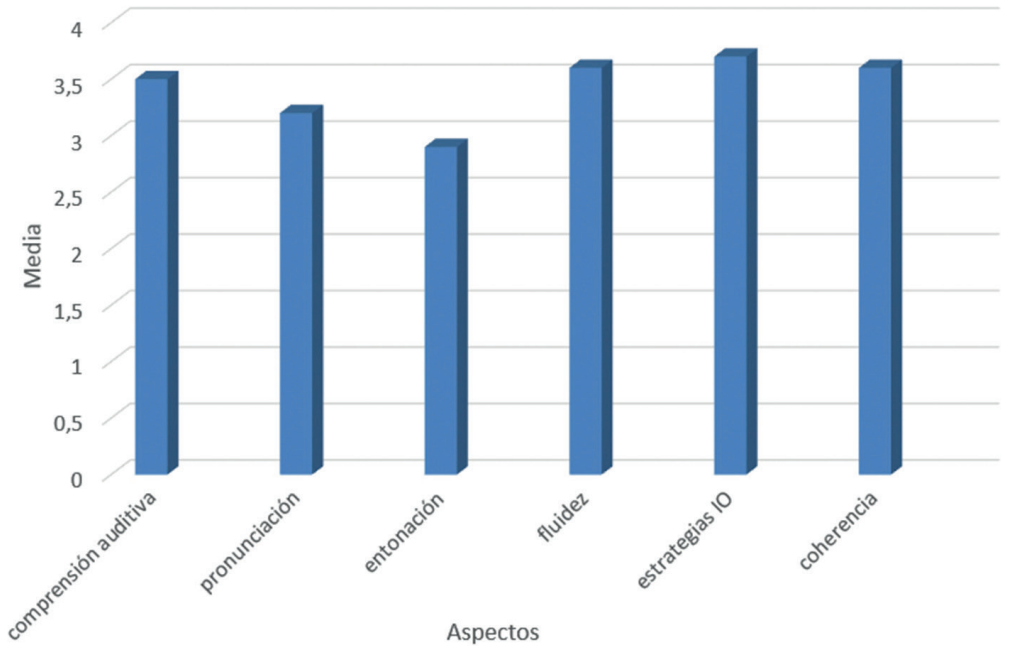

Figura 3. Percepción de los aprendices sobre los aspectos de la expresión oral mejorados con la IDAM.

En cuanto al segundo ítem, la efectividad de las diferentes tareas para alcanzar el objeto de aprendizaje, los estudiantes debieron valorar el grado en que cada una de las tareas contribuyó a la mejora de la IO, como muestra la tabla 2.

Tabla 2. Opiniones de los sujetos sobre el grado en que cada una de las tareas contribuyó a la mejora de la IO. Con una escala de 1 a 5 donde $1=$ muy bajo y $5=$ muy alto

\begin{tabular}{|l|c|}
\hline \multicolumn{1}{|c|}{ Tareas } & Media \\
\hline Escuchar, comparar las charlas 1 y 2 y dejar los comentarios sobre ellas en FB. & 3,85 \\
\hline Leer/escuchar la explicación sobre las estrategias de IO en VoiceThread. & 3,85 \\
\hline Escuchar, repetir y grabar los marcadores léxicos en VoiceThread. & 3,38 \\
\hline Practicar las frases con Glosboken. & 3,73 \\
\hline Practicar las frases con Quia. & 3,42 \\
\hline Analizar las estrategias de interacción usadas en las tres charlas y escribir en la guía digital. & 3,77 \\
\hline Realizar charlas cortas similares y grabarlas en VoiceThread. & 4,08 \\
\hline Realizar una entrevista con un joven colombiano vía internet. & 2,92 \\
\hline Crear una animación con GoAnimate. & 2,69 \\
\hline
\end{tabular}

Como podemos observar, las tareas señaladas como más efectivas son las siguientes: en primer lugar, la tarea de realizar y grabar charlas similares a las charlas modelo; en segundo 
lugar, la tarea de escuchar y comparar las charlas introductorias para luego dejar los comentarios sobre ellas en Facebook, conjuntamente con la tareas de leer y/o escuchar la explicación sobre estrategias de IO; y, en un tercer lugar, la tarea de analizar las estrategias de IO utilizadas en las charlas modelo. Cabe señalar que la tarea valorada como más efectiva es de carácter altamente comunicativo, mientras las que ocupan el segundo lugar son dos tareas posibilitadoras que permiten aproximarse al nuevo conocimiento desde las nociones previas de los aprendices y desde la explicación de un experto. La tarea que ocupa el tercer lugar es también posibilitadora pero de carácter metacognitivo, por cuanto le brinda al aprendiz la posibilidad de hacer consciente el conocimiento adquirido, al analizar las estrategias de IO utilizadas en las charlas modelo. Esto permite concluir que los aprendices encuentran más efectivas las tareas de carácter comunicativo, lo cual está totalmente en concordancia con los postulados del enfoque metodológico que subyace a la intervención didáctica.

Pasando ahora al tercer ítem, los logros de la IDAM, podemos señalar que los aprendices percibieron que esta forma de trabajo fue interesante y enriquecedora. Esto se puede observar en la figura 4 donde se muestra el valor de la media, en una escala Likert de 1 a 5 , para las siguientes afirmaciones propuestas a los alumnos acerca de la IDAM:

1. Ha aumentado mi motivación para aprender español.

2. Ha incrementado la confianza en mí mismo para comunicarme oralmente en español.

3. Desarrollarla fue divertido.

4. Fue interesante y enriquecedora.

5. Fue de fácil desarrollo.

6. Estuvo bien organizada.

7. Me gustaría trabajar de esta forma nuevamente.

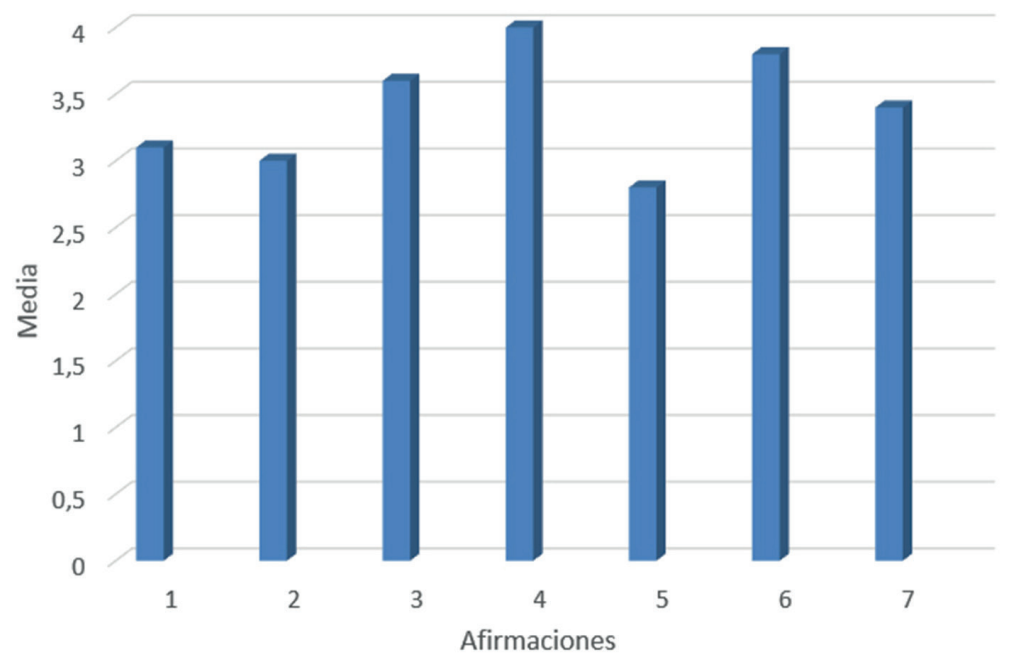

Figura 4. Valores medios asignados a diferentes afirmaciones sobre la IDAM 
Como se puede observar, los estudiantes muestran una actitud positiva hacia el uso de la IDAM, ya que asignaron un valor relativamente alto a varias de las afirmaciones propuestas, siendo la mejor valorada la numero 4: «fue interesante y enriquecedora», con un promedio de 4.00. En segundo lugar valoraron la afirmación: «estuvo bien organizada», con un promedio de 3.84 y, en tercer lugar, la afirmación «fue divertido desarrollarla», con 3.60. Nótese que la afirmación que obtuvo una media más baja $(2,88)$ fue la relacionada con la facilidad de su desarrollo (5). Esto se puede explicar a partir de la poca familiaridad que los estudiantes tenían con esta metodología en el momento de aplicar la IDAM.

Efectivamente, por medio del cuestionario y de las entrevistas realizadas se pudo establecer que a pesar de que los estudiantes participantes podrían considerarse como nativos digitales (Prensky, 2005), esta fue su primera experiencia con MALL. En este sentido, los participantes señalaron como uno de los factores que les resultó más novedoso y que, por lo tanto, les generó mayor dificultad, el hecho de tener que interactuar con la profesora solamente a través de los medios digitales dispuestos para ese objetivo, como el grupo de Facebook. Sin embargo, esta misma situación también fue identificada como positiva debido a que les obligó a solucionar por sí mismos los problemas encontrados.

Las entrevistas también permitieron concluir que, en general, los aprendices valoraron la experiencia como muy enriquecedora y positiva. Dentro de los factores que contribuyeron a esto señalaron la posibilidad de regular su propio aprendizaje al ser ellos los que tenían el control sobre los procesos y las tareas por realizar, así como sobre el ritmo de aprendizaje.

También dijeron haber asumido más responsabilidad en la realización de las tareas, debido al hecho de que serían compartidas con los compañeros de clase. Esta colaboración fue considerada, además, como benéfica para el aprendizaje, pues el hecho de ver las producciones de los otros estudiantes, analizarlas y compararlas con las propias, les proporcionó nuevas ideas y les permitió hacer una autoevaluación de sus propias producciones. Todo esto contribuyó a la construcción del aprendizaje.

Por otra parte, llama la atención el hecho de que, a pesar de no haber señalado la posibilidad de entrevistar en línea a un hablante nativo como una de las tareas más útiles, sí la identificaron como la tarea más motivadora de todas las propuestas en la IDAM, por cuanto les dio la posibilidad de aplicar los conocimientos adquiridos a una situación real de comunicación. La baja percepción de utilidad pudo deberse a las dificultades técnicas encontradas al realizar la entrevista o al hecho de que no se logró un alto grado de interacción, como muchos estudiantes lo manifestaron en las entrevistas grupales.

Los aprendices manifestaron también que percibieron las tareas incluidas en la IDAM como variadas y divertidas, lo cual contribuyó a que las llevaran a cabo con un mayor entusiasmo y dedicación. Igualmente, encontraron que mediante la realización de las tareas tuvieron la oportunidad de identificar sus debilidades y necesidades de aprendizaje; también la posibilidad de activar las estrategias de IO, pues aunque las habían usado al comunicarse en lengua materna, no eran conscientes de su existencia y por tanto no habían considerado usarlas al comunicarse en LE. Percibieron, además, que las diferentes tareas mediante las cuales ensayaron las estrategias de IO contribuyeron a aumentar su autoconfianza para hablar en la lengua objeto. 
Los estudiantes evaluaron la página de Facebook y su la función de chat como un recurso útil y divertido porque les brindó la oportunidad de ver las creaciones de los compañeros de clase; además, porque les brindó la posibilidad de estar en contacto permanente con los otros participantes y con la profesora, incluso fuera de los horarios de clase y fuera de la escuela. Algunos de ellos dijeron haber usado estos recursos durante el fin de semana para resolver dudas acerca de la entrevista con el hablante nativo, por ejemplo. En general, encontraron que fue una forma de comunicación bastante efectiva, aunque para algunos resultó molesta, como se explica más adelante.

Otra ventaja fue la de tener acceso permanente a información escrita en la lengua objeto ya que podían revisarla cuantas veces quisieran e incluso hacer uso de otras herramientas digitales, como los traductores, para entender el mensaje. Percibieron que todos estos factores contribuyeron bastante al proceso de aprendizaje.

Por último, en lo referente a la forma de realizar la evaluación final, es decir, mediante la grabación de un diálogo guiado en VoiceThread, la mayoría de los participantes manifestó preferir esta forma al modo tradicional de hablar en vivo delante del profesor. Estos estudiantes argumentaron su respuesta explicando que al grabar se sintieron menos nerviosos y tuvieron más oportunidad de prepararse. Además, se sintieron más seguros por cuanto ya habían realizado tareas similares durante el desarrollo de la IDAM.

Pasando ahora a las dificultades de la metodología de trabajo usando dispositivos y recursos móviles, los aprendices señalaron como la principal de ellas el riesgo que existe, en estos medios, de distorsión y ambigüedad comunicativas. En este sentido, dijeron preferir la comunicación cara a cara con el profesor por permitir una interacción más directa, que proporciona un mayor grado de seguridad sobre la comprensión de los contenidos y de las instrucciones.

Algunos también señalaron los problemas de funcionamiento de la tecnología como una desventaja porque causó desmotivación, les hizo perder el ritmo del trabajo y retrasó la realización de las tareas. Asimismo, algunos de los aprendices, si bien una minoría, manifestaron haber tenido dificultades para autorregular el manejo del tiempo y de la realización de las tareas. Estos estudiantes experimentaron problemas de concentración y de toma de decisiones; a consecuencia de ello experimentaron estrés ya que tuvieron que realizar las tareas en muy poco tiempo. Además, este grupo de estudiantes percibió como negativo el hecho de recibir información en Facebook durante el tiempo extraescolar. Dijeron sentirse presionados y estresados por esto. Para estos aprendices tampoco resultó atractiva la grabación de la tarea final en VoiceThread; ya que declararon, nuevamente, sentir estrés, haber olvidado todo y haber quedado insatisfechos con el resultado.

También se pudieron identificar dificultades relacionadas con la realización de la entrevista al hablante nativo. Estos inconvenientes se debieron a factores tecnológicos y logísticos, al manejo de la lengua objeto y a factores afectivos. Desde el punto de vista técnico, las dificultades se debieron a problemas de acceso a internet y de funcionamiento de los recursos tecnológicos usados por los hablantes nativos; los problemas logísticos se presentaron principalmente por la diferencia horaria entre los participantes suecos y sus 
pares hispanohablantes ${ }^{5}$. Con respecto al manejo de lengua, se debieron al idiolecto utilizado por el entrevistado y la velocidad de su elocución.

Las dificultades relacionadas con factores afectivos tuvieron que ver con la ausencia de contacto previo con el hablante entrevistado. Esto generó nerviosismo y tensión en muchos de los participantes. Cabe anotar que, debido a las diferencias horarias, varios de los aprendices no lograron cumplir con esta tarea; sin embargo, manifestaron que el solo hecho de tener que prepararla contribuyó, como se mencionó antes, a que realizaran las tareas con mayor interés y a que mejoraran en el manejo de las estrategias de IO.

\section{CONCLUSIONES}

El análisis antes presentado permite concluir que los aprendices percibieron que la IDAM fue una intervención didáctica marcada ante todo por tres características: efectividad, innovación y entretenimiento.

La efectividad estuvo dada ante todo por el tipo de tareas que fueron consideradas variadas, dinámicas y comunicativas; también como tareas que permitieron la repetición y el ensayo de los nuevos conocimientos y el contacto con material audiovisual auténtico. Los estudiantes encontraron especialmente interesante la interacción con el hablante nativo que, si bien implicó dificultades y en algunos casos frustración, fue una tarea enriquecedora y novedosa. Los aprendices percibieron, además, que la mayoría de las tareas cumplieron el objetivo para el cual fueron diseñadas: desarrollar la destreza de IO.

Cabe destacar el hecho de que su atención estuvo más centrada en el desarrollo de las destrezas mismas y no en la memorización de sus marcadores léxicos. Esto es revelador si se tiene en cuenta que el componente léxico sigue siendo uno de los más priorizados al crear aplicaciones móviles para el aprendizaje de las lenguas en los últimos años (Sung, Y. T. et al., 2015).

Otro factor que contribuyó a percibir que la IDAM fue efectiva tuvo que ver con su función como facilitadora y/o activadora de procesos relevantes para el aprendizaje de lenguas, tales como la concienciación de fenómenos y usos de la lengua (metacognición), el fortalecimiento de la autoconfianza y la autorregulación del aprendizaje; principios todos contemplados en el constructivismo y la psicología social, modelos pedagógicos que subyacen a este estudio (Vigotsky, 1967; Gardner, 1985; Ellis, 1985). El carácter de los componentes valorados por los estudiantes como más efectivos también habla a favor de los enfoques didácticos de su diseño y desarrollo (el trabajo por tareas y el enfoque comunicativo) por cuanto coinciden con muchos de sus principios básicos.

En cuanto al carácter innovador de la IDAM, resultó evidente que los estudiantes nunca antes habían usado esta modalidad de aprendizaje y esto generó muchos retos; sin embargo, también despertó su interés e incrementó su motivación.

${ }^{5}$ Los pares hispanohablantes fueron jóvenes habitantes de Cajicá, una pequeña población colombiana. 
Uno de los factores que contribuyó claramente a que los estudiantes percibieran la IDAM como novedosa fue el uso de tecnología muy cercana a ellos, como los dispositivos móviles y las redes sociales. Pero lo que generó más impacto fue la ausencia física de la profesora, puesto que los estudiantes asistían a una escuela de modalidad presencial y nunca habían participado en una situación similar de aprendizaje. Este hecho tuvo mayores implicaciones en lo relacionado con la autorregulación del aprendizaje y con la interacción alumnos-profesor y alumnos-alumnos.

Respecto a lo primero, los estudiantes valoraron positivamente la posibilidad de tener más control sobre su propio proceso de aprendizaje en términos de tiempo, esfuerzo, cronología de desarrollo, solución de problemas y toma de decisiones y responsabilidad. En cuanto a la interacción con el profesor, si bien inicialmente resultó frustrante para muchos, a largo plazo se normalizó y los estudiantes empezaron a ver las ventajas de la interacción a través de medios digitales: mayor individualización en el tratamiento de las preguntas, retroalimentación más frecuente y oportunidad de realizar consultas en cualquier tiempo y espacio, por ejemplo.

Por su parte, la interacción alumnos-alumnos a través de los medios digitales fue percibida como muy positiva y enriquecedora, ante todo debido a la posibilidad de compartir ideas y aprender mutuamente del trabajo realizado. Esta interacción constituyó un regulador de desempeño por cuanto los aprendices sintieron mayor compromiso en la realización de las tareas, al saber que podrían ser vistas por todos los participantes del grupo. Todos estos elementos influyeron para que los estudiantes asumieran una actitud más positiva y comprometida. Se generó así una nueva dinámica de aprendizaje.

El factor lúdico fue otra característica identificada a partir de las percepciones de los aprendices acerca de la IDAM. En efecto, fueron muchas las opiniones que describieron varias de las tareas como divertidas y por tanto, facilitadoras del aprendizaje. Esta propiedad de la IDAM se puede considerar como un valor añadido por cuanto es uno de los aspectos señalados como relevantes en el modelo comunicativo de aprendizaje de las lenguas, en la medida en que está relacionado con factores afectivos y motivacionales (MCER, 2002).

También es útil e importante referirse en estas conclusiones a los hechos señalados por los estudiantes como dificultades en el proceso de aprendizaje desarrollado con la IDAM. En primer lugar, resulta significativo el hecho de que dichos inconvenientes son pocos y de dimensiones manejables, si se tiene en cuenta que los sujetos participantes nunca habían tenido una experiencia del mismo tipo o similar. Esto indica que los aprendices se adaptaron rápida y eficientemente a las exigencias del nuevo método. Estas dificultades estuvieron relacionadas principalmente con tres aspectos, en orden de frecuencia: obtención de ayuda por parte de la profesora, comprensión de las instrucciones y problemas relativos a la tecnología utilizada. Si bien estos problemas generaron cierta frustración, y en ocasiones irritación en algunos de los estudiantes, todos fueron superados y no llegaron a ser un verdadero obstáculo para el aprendizaje. Sin embargo, constituyen un valioso punto de referencia para el desarrollo de futuras intervenciones didácticas similares.

Esperamos que la experiencia descrita en estas páginas contribuya a incorporar los dispositivos y recursos móviles al aula de segundas lenguas y de lenguas extranjeras, de una manera responsable e innovadora y, ante todo, que anime a otros profesores e investigadores 
a seguir indagando sobre las posibilidades que ofrece el aprendizaje móvil para desarrollar los diferentes componentes de la destreza de expresión oral.

Creemos que el mayor aporte de este estudio radica en que aborda un aspecto que no se había estudiado de manera específica hasta el momento: la aplicación de MALL al desarrollo de las estrategias de IO, factor relevante incluso en los niveles iniciales de aprendizaje de una LE. Tal y como manifestaron algunos de los participantes de esta experiencia, el poder desarrollar una charla fluida, aunque simple y corta, incrementa en alto grado la motivación y la autoconfianza, aspectos definitivos para el aprendizaje de LE.

Consideramos, por último, que la pedagogía en general, y la didáctica de las lenguas en particular, está atravesando por un momento crucial en el que postulados de vieja escuela pero aún válidos, como los vigotskianos, los cognitivistas y los constructivistas, encuentran, como nunca antes, modos y medios para ser aplicados exitosamente a los procesos de aprendizaje. Corresponde a profesores e investigadores indagar sobre las mejores formas de hacerlo.

\section{REFERENCIAS BIBLIOGRÁFICAS}

Ahn, Tae Youn \& Lee, Sangmin-Michelle (2015): «User experience of a mobile speaking application with automatic speech recognition for EFL learning». British Journal of Educational Technology, doiI: 10.1111/bjet.12354.

AFSHAR, VALA (2014): 50 incredible WiFi tech statistics that businesses must know. Recuperado el 16 de enero de 2016 de http://www.huffingtonpost.com/vala-afshar/50-incredible-wifi-techs_b_4775837.html.

Bordon, Teresa. (2008). «La evaluación de la expresión oral y la comprensión auditiva». En Sánchez Lobato, Jesús y Santos Gallardo, Isabel (coord.), Vademécum para la formación de profesores. Enseñar español como segunda lengua (L2)/Lengua extranjera (LE). Madrid, SGEL, págs. 983-1001

Breen, Michael P. (1987): «Learner contributions to task design». Candlin, Christopher Y Murphy, Dermot (Eds), Language Learning Tasks. Englewood Cliffs: Prentice Hall, págs. 23-46.

Burston, Jack (2014): «The reality of MALL: Still on the fringes». CALICO Journal, 31 (1), págs. 103-125. doi:10.11139/cj.31.1.103-125.

Canale, Michel \& Swain, Merril (1980): «Theoretical bases of communicative approaches to second language teaching and testing». Applied Linguistics (1), págs. 1-47.

CANDlin, Christopher N. (1990): «Hacia la enseñanza de lenguas basada en tareas». Comunicación, Lenguaje y Educación, 7 (8), págs. 33-53.

Consejo de Europa (2002): Marco Común Europeo De Referencia Para las Lenguas: Aprendizaje, Enseñanza, Evaluación. Recuperado el 25 de junio de 2015 de http://cvc.cervantes.es/ensenanza/biblioteca_ele/marco/.

Deegan, Robin. (2015): «Complex mobile learning that adapts to learners' cognitive load». International Journal of Mobile and Blended Learning, 7. doi: 0.4018/IJMBL, págs. 13-24. Recuperado el 17 enero de 2016 de: http:// www.igiglobal.com/viewtitlesample.aspx?id=124167\&ptid=118568\&t=Complex\%20Mobile\%20Learning\%20 that $\% 20$ Adapts $\% 20$ to\%20Learners\%27\%20Cognitive\%20Load.

Demouy, Valérie \& Kukulska-Hulme, Agnes (2010):»On the spot: using mobile devices for listening and speaking practice on a French language program». Open Learning: The Journal of Open and Distance Learning, 25(3), págs. 217-232.

Ellis, RoD (1985): Understanding Second Language Acquisition. Oxford, Oxford University Press.

EstaIRE, SHeIla (2004): «La programación de unidades didácticas a través de tareas». Recuperado el 28 de junio de 2015: http://www.nebrija.es/espanolparainmigrantes/flash/ensenar/PDF/articulo-tareas.pdf.

Gardner, Robert C. (1985): Social Psychology and Second Language Learning: the Roles of Attitudes and Motivation. Ontario, Edward Arnold. 
Gromiк, Nicolas A. (2015): «The effect of smartphone video camera as a tool to create digital stories for English learning purposes». Journal of Education and Learning, 4 (4), pág. 64.

Hughes, Rebecca (2002): Teaching and researching - Speaking. Great Britain, Pearson Education.

Ibáñez Moreno, Ana; Vermeulen, Anna \& Jordano De La Torre, María (2016): «Diseño y evaluación de VISP, una aplicación móvil para la práctica de la competencia oral». RIED: Revista Iberoamericana de Educación a Distancia, 19 (1), págs. 63-81.

Ibáñez Moreno, Ana \& Vermeulen, Anna. (En prensa): «Profiling a MALL app for English oral practice. A case study.». Journal of Universal Computer Science.

Kukulska-Hulme, Agnes; Norris, Lucy \& Donohu, Jim (2015): «Mobile pedagogy for English language teaching: A guide for teachers». ELT Research Papers 14.07 British Council, págs. 1-41. Recuperado el 5 de febrero de 2015 de http://englishagenda.britishcouncil.org/sites/ec/files/E485\%20Mobile\%20pedagogy\%20 for\%20ELT_FINAL_v2.pdf.

Lazaraton, AnNe (2001): «Teaching Oral Skills». Teaching English as a second foreign language. III ed. Boston, Heinle and Heinle, págs. 103-115.

Littlewood, William (1981): La enseñanza comunicativa de idiomas. Cambridge, Cambridge University Press.

Melero Abadia, Pilar (2008): «De los programas nocional-funcionales a la enseñanza comunicativa». En Sánchez Lobato, Jesús y Santos Gallardo, Isabel (coord.), Vademécum para la formación de profesores. Enseñar español como segunda lengua (L2)/Lengua extranjera (LE). Madrid, SGEL, págs. 689-714.

Moladoust, Elahe (2014): «EFL teacher's and learners' perspectives towards ATODJ: A case of CMC and MALL». International Journal of Computer - Assisted Language Learning and Teaching, 4 (2), págs. 29-45. doi:10.4018/ijcallt.2014040103.

Nunan, David (1989): Designing Tasks for the Communicative Classroom. Cambridge, Cambridge University Press.

Pegrum, Mark (2014): Mobile Learning: Languages, Literacies and Cultures. Basingstoke, Palgrave Macmillan. doi:10.1057/9781137309815.

Prabhu, Neiman Stern (1987): Second Language Pedagogy. Oxford, Oxford University Press.

Prensky, Marc (2005): «Listen to the natives». Educational Leadership, 63 (4), pág. 8.

Richards, JaCk \& Rodgers, Theodore S. (2010): Approaches and Methods in Language Teaching. Cambridge, Cambridge University Press.

Sharples, Mike; Taylor, Josie \& Vavoula, Giasemi (2007): «A Theory of Learning for the Mobile Age». Andrews, Richard \& Haythornthwaite, Caroline (Eds.), The Sage Handbookof Elearning Research. London, Sage, págs. 221-247.

Shuler, Carly; Winters, Niall \& West, Mark (2013): El futuro del aprendizaje móvil. Implicaciones para la planificación y formulación de políticas. París, UNESCO

Sung, Yao-Ting; Chang, Kuo-En \& YAng, Je-Ming (2015): How effective are mobile devices for language learning? A meta-analysis, educational research review, doi: 10.1016/j.edurev.2015.09.001.

TAYlor, Josie (2005): Digital Technologies and Cognitive Development. Recuperado el 20 de noviembre de 2014 de: http://www.oecd.org/edu/ceri/38360564.pdf.

Viberg, Olga \& Grönlund, ÅKe (2013): «Systematizing the field of mobile assisted language learning». International Journal of Mobile and Blended Learning, 5(4), págs. 72-90. Recuperado el 12 de julio de 2016 de http://www.igi-global.com/viewtitlesample.aspx?id=99681.

Vigotsky, Lev. S. (1978): Mind in society: The development of higher psychological processes. Harvard, Harvard University Press.

Wilkins, David Arthur (1976): Notional Syllabuses: A Taxonomy and its Relevance to Foreign Language Curriculum Development. Oxford, Oxford University Press.

Willis, David \& Willis, Jane (2007): Doing Task-based Teaching. Oxford, Oxford University Press. 